Document downloaded from:

http://hdl.handle.net/10251/51870

This paper must be cited as:

Bru García, R.; Cantó Colomina, R.; Soto, Ricardo L. ; Urbano Salvador, AM. (2012). A Brauer's theorem and related results. Central European Journal of Mathematics. 10(1):312321. doi:10.2478/s11533-011-0113-0.

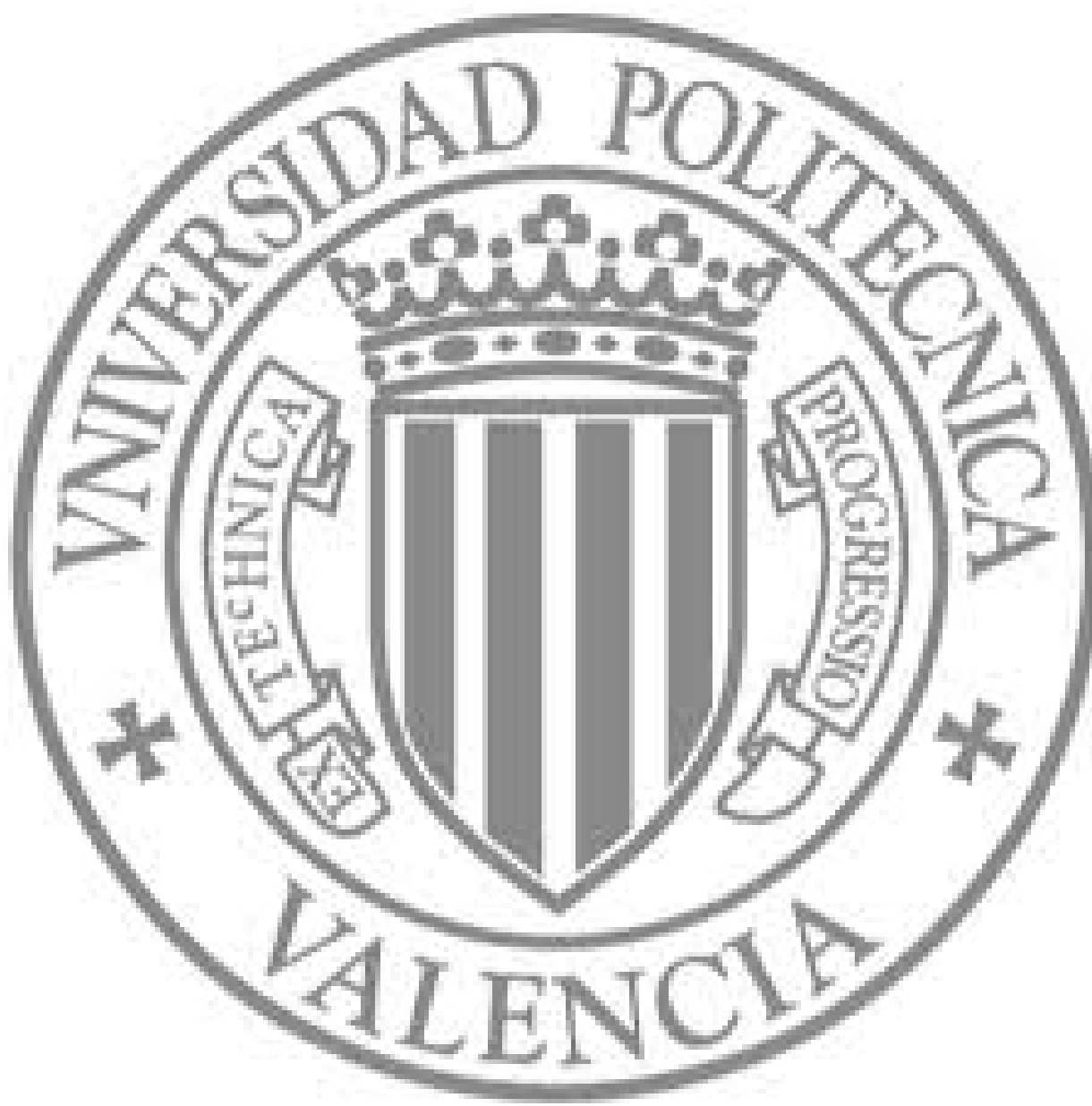

The final publication is available at

http://dx.doi.org/10.2478/s11533-011-0113-0

Copyright Springer Verlag (Germany) 


\title{
A Brauer's theorem and related results
}

\author{
R. Bru* R. Cantó* $\quad$ R. L. Soto ${ }^{\dagger} \quad$ A.M. Urbano*
}

\begin{abstract}
Given a square matrix $A$, a Brauer's theorem [Limits for the characteristic roots of matrices IV: Applications to stochastic matrices, Duke Math. J. 19 (1952), 75-91] shows how to modify one single eigenvalue of $A$ via a rank-one perturbation without changing any of the remaining eigenvalues. Older and newer results can be considered in the framework of the above theorem. In this paper, we present its application to stabilization of control systems, including the case when the system is noncontrollable. Other applications presented are related to the Jordan form of $A$ and Wielandt's and Hotelling's deflations. An extension of aforementioned Brauer's result, Rado's theorem, shows how to modify $r$ eigenvalues of $A$ at the same time via a rank- $r$ perturbation without changing any of the remaining eigenvalues. The same results considered by blocks can be put into the block version framework of the above theorem.
\end{abstract}

Keywords: eigenvalues, pole assignment problem, controllability, low rank perturbation, deflation techniques.

MSC: 15A18, 93D15.

\section{Brauer's theorem}

The relationship among the eigenvalues of an arbitrary matrix and the updated matrix by a rank-one additive perturbation was established by A. Brauer [1]. We will refer to this result as Brauer's Theorem. It turns out that this result is related to older and well-known results on Wielandt's and Hotelling's deflations techniques [10]. Brauer's Theorem finds its application also in the eigenvalue localization problem of control theory (see [5]) and in stabilization of control systems. Perfect [7] applied an extension of Brauer's result, Rado's theorem, to construct nonnegative matrices with a prescribed spectrum.

In the first part of the paper (Sections 1 and 2), we give results that can be considered in a common framework of Brauer's Theorem as applications of it. A good introduction on the Brauer result and its application to the nonnegative

\footnotetext{
*Institut de Matemàtica Multidisciplinar, Universitat Politècnica de València, 46022 València, Spain. (rbru@mat.upv.es, rcanto@mat.upv.es, amurbano@mat.upv.es)

${ }^{\dagger}$ Departamento de Matemáticas, Universidad Católica del Norte, 1280 Antofagasta, Chile. (rsoto@ucn.cl)
} 
inverse eigenvalue problem can be followed in [9] where Rado's theorem is given. Rado's theorem is considered in the second part of this paper (Sections 3 and 4) and applied to obtain a block version of deflation results.

Throughout the paper, we assume that all sets of eigenvalues of a matrix are feasible in the corresponding field (i.e., closed under complex conjugation in the real field).

Theorem 1 ([7, 9, Brauer's Theorem]) Let $A$ be an arbitrary $n \times n$ matrix with eigenvalues $\sigma(A)=\left\{\lambda_{1}, \lambda_{2}, \ldots, \lambda_{n}\right\}$. Let $x_{k}$ be an eigenvector of $A$ associated with the eigenvalue $\lambda_{k}$, and let $q$ be any $n$-dimensional vector. Then the matrix $A+x_{k} q^{T}$ has eigenvalues $\left\{\lambda_{1}, \ldots, \lambda_{k-1}, \lambda_{k}+x_{k}^{T} q, \lambda_{k+1}, \ldots, \lambda_{n}\right\}$.

Let $\lambda_{k}$ be an eigenvalue of $A$ with a Jordan chain of length 1 and let $q$ be a vector orthogonal to the remaining eigenvectors of $A$. Then, it can be seen that the Jordan structures of $A$ and $A+x_{k} q^{T}$ are the same.

The relationships among the right eigenvectors of $A$ and $A+x_{k} q^{T}$ are given in the following result [8].

Proposition 1 Let $A$ be an arbitrary $n \times n$ matrix with eigenvalues $\sigma(A)=\left\{\lambda_{1}\right.$, $\left.\lambda_{2}, \ldots, \lambda_{n}\right\}$. Let $x_{i}$ be an eigenvector of $A$ associated with the eigenvalue $\lambda_{i}$, $1 \leq i \leq n$. Let $q$ be any $n$-dimensional vector and let $\mu_{k}=\lambda_{k}+x_{k}^{T} q$, with $\mu_{k} \neq \lambda_{i}, i=1,2, \ldots, n$. Then, $x_{k}$ is an eigenvector of the matrix $A+x_{k} q^{T}$ associated with the eigenvalue $\mu_{k}=\lambda_{k}+x_{k}^{T} q$, and the eigenvectors of $A+x_{k} q^{T}$ associated with $\lambda_{i}, i \neq k$, are:

$$
w_{i}=x_{i}-\frac{q^{T} x_{i}}{\mu_{k}-\lambda_{i}} x_{k}
$$

However, the changes of the left eigenvectors of $A$ and $A+x_{k} q^{T}$ are inverse as we can see in the next result for a diagonalizable matrix $A$.

Proposition 2 Let $A$ be a diagonalizable $n \times n$ matrix with eigenvalues $\sigma(A)=$ $\left\{\lambda_{1}, \lambda_{2}, \ldots, \lambda_{n}\right\}$. Let $l_{i}^{T}$ be a left eigenvector of $A$ corresponding to $\lambda_{i}, 1 \leq$ $i \leq n$. Let $q$ be any $n$-dimensional vector and let $\mu_{k}=\lambda_{k}+x_{k}^{T} q$, with $\mu_{k} \neq \lambda_{i}$, $i=1,2, \ldots, n$. Then, the left eigenvectors of $A+x_{k} q^{T}$ corresponding to $\lambda_{i}$, $i \neq k$, are $r_{i}^{T}=l_{i}^{T}$, and the left eigenvector of $A+x_{k} q^{T}$ corresponding to $\mu_{k}$ is:

$$
r_{k}^{T}=l_{k}^{T}+\sum_{\substack{i=1 \\ i \neq k}}^{n} \frac{q^{T} x_{i}}{\mu_{k}-\lambda_{i}} l_{i}^{T} .
$$

Proof: Since $l_{i}^{T}, i \neq k$, is a left eigenvector of $A$ corresponding to $\lambda_{i}$, we have $l_{i}^{T}\left(A-\lambda_{i} I\right)=0, i \neq k$. So, $\left\langle l_{i}, x_{k}\right\rangle=0$, for all $i \neq k$, and

$l_{i}^{T}\left(A+x_{k} q^{T}-\lambda_{i} I\right)=l_{i}^{T}\left(A-\lambda_{i} I\right)+l_{i}^{T}\left(x_{k} q^{T}\right)=0+\left(l_{i}^{T} x_{k}\right) q^{T}=\left\langle l_{i}, x_{k}\right\rangle q^{T}=0$. 
Hence $l_{i}^{T}, i \neq k$, is a left eigenvector of $A+x_{k} q^{T}$ corresponding to $\lambda_{i}: r_{i}^{T}=l_{i}^{T}$, $i \neq k$. Since

$$
\begin{aligned}
& \left(l_{k}^{T}+\sum_{\substack{i=1 \\
i \neq k}}^{n} \frac{q^{T} x_{i}}{\mu_{k}-\lambda_{i}} l_{i}^{T}\right)\left(A+x_{k} q^{T}\right) \\
& =l_{k}^{T} A+l_{k}^{T} x_{k} q^{T}+\sum_{i=1}^{n} \frac{q^{T} x_{i}}{\mu_{k}-\lambda_{i}} l_{i}^{T} A+\sum_{i=1}^{n} \frac{q^{T} x_{i}}{\mu_{k}-\lambda_{i}} l_{i}^{T} x_{k} q^{T} \\
& i \neq k \quad i \neq k \\
& =\lambda_{k} l_{k}^{T}+q^{T}+\sum_{i=1}^{n} \frac{q^{T} x_{i}}{\mu_{k}-\lambda_{i}} \lambda_{i} l_{i}^{T} \\
& i \neq k \\
& =\lambda_{k} l_{k}^{T}+\sum_{i=1}^{n} \frac{q^{T} x_{i}}{\mu_{k}-\lambda_{i}} \lambda_{i} l_{i}^{T}+q^{T}(\underbrace{x_{1} l_{1}^{T}+x_{2} l_{2}^{T}+\cdots+x_{n} l_{n}^{T}}_{I}) \\
& i \neq k \\
& =(\lambda_{k}+\underbrace{q^{T} x_{k}}_{\mu_{k}-\lambda_{k}}) l_{k}^{T}+\sum_{\substack{i=1 \\
i \neq k}}^{n}\left(\frac{q^{T} x_{i}}{\mu_{k}-\lambda_{i}} \lambda_{i}+q^{T} x_{i}\right) l_{i}^{T} \\
& =\mu_{k} l_{k}^{T}+\sum_{\substack{i=1 \\
i \neq k}}^{n} \frac{q^{T} x_{i}}{\mu_{k}-\lambda_{i}} \mu_{k} l_{i}^{T}=\mu_{k}\left(l_{k}^{T}+\sum_{\substack{i=1 \\
i \neq k}}^{n} \frac{q^{T} x_{i}}{\mu_{k}-\lambda_{i}} l_{i}^{T}\right)=\mu_{k} r_{k}^{T}, \\
& r_{k}^{T}=l_{k}^{T}+\sum_{\substack{i=1 \\
i \neq k}}^{n} \frac{q^{T} x_{i}}{\mu_{k}-\lambda_{i}} l_{i}^{T}
\end{aligned}
$$

\section{Related results}

In this section we show that Brauer's Theorem [1] can be used to prove different results. For instance, to examine existence and convergence of the Page Rank Power Method, a stochastic matrix is updated by a rank-one matrix to construct the Google matrix. The relationship between the spectrum of both matrices is 
given in [6, Theorem 5.1]. The same result can be obtained as a corollary of Brauer's Theorem 1 applied to the matrix $\alpha A$ and the vector $q=(1-\alpha) v$.

\subsection{Deflation techniques}

In 1944 Wielandt presented a deflation method for general matrices shifting one eigenvalue to zero (see [10]). Application of Brauer's Theorem 1 with a vector $q$ such that $q^{T} x_{k}=-\lambda_{k}$ immediately gives this result.

Corollary 1 (Wielandt's deflation) Let assumptions of Theorem 1 hold with $q$ being any vector such that $q^{T} x_{k}=-\lambda_{k}$, then the matrix $A+x_{k} q^{T}$ has the eigenvalues $\left\{\lambda_{1}, \ldots, \lambda_{k-1}, 0, \lambda_{k+1}, \ldots, \lambda_{n}\right\}$.

Remark 1 If $A$ is symmetric, then $A$ is diagonalizable and we can choose an orthogonal matrix $X=\left[\begin{array}{llll}x_{1} & x_{2} & \ldots & x_{n}\end{array}\right]$ made of the eigenvectors of $A$. In this case the matrix $B=A+\left(\mu_{k}-\lambda_{k}\right) x_{k} x_{k}^{T}$ is symmetric (diagonalizable) and it can be verified that the eigenvectors of $B$ associated with $\lambda_{i}, i \neq k$, are the eigenvectors of $A$ associated with $\lambda_{i}, i \neq k$.

The above result contains an older technique due to Hotelling, established in 1933, for symmetric matrices that can be extended to nonsymmetric matrices.

Corollary 2 (Hotelling's deflation) Let assumptions of Theorem 1 hold. (i) (Symmetric case.) Let $A$ be symmetric. Then the symmetric matrix $A-$ $\lambda_{k} x_{k} x_{k}^{T}$ has the eigenvalues $\left\{\lambda_{1}, \lambda_{2}, \ldots, \lambda_{k-1}, 0, \lambda_{k+1}, \ldots, \lambda_{n}\right\}$, provided that $x_{k}^{T} x_{k}=1$.

(ii) (Nonsymmetric case.) Let $l_{k}$ be the left eigenvector of $A$, with $l_{k}^{T} x_{k}=1$. Then the matrix $A-\lambda_{k} x_{k} l_{k}^{T}$ has the eigenvalues $\left\{\lambda_{1}, \ldots, \lambda_{k-1}, 0, \lambda_{k+1}, \ldots, \lambda_{n}\right\}$.

Proof: Apply Brauer's Theorem 1 with a vector $q=-\lambda_{k} x_{k}$ in the symmetric case and $q=-\lambda_{k} l_{k}$ in the nonsymmetric case.

\subsection{Pole assignment of SISO systems}

Another application Brauer's Theorem 1 finds for single-input single-output (SISO) linear time invariant control systems when the system given by a pair $(A, b)$ is not completely controllable. Given a SISO system we use a state feedback to place the poles of the closed-loop system at specified points in the complex plane. More precisely, the pole placement problem states as follows:

Consider a pair $(A, b)$. Let $\sigma(A)=\left\{\lambda_{1}, \lambda_{2}, \ldots, \lambda_{n}\right\}$ and let $\mu_{k}$ be a number. Under what conditions on $(A, b)$ does there exist a vector $f$ such that the spectrum of the closed-loop system $A+b f^{T}, \sigma\left(A+b f^{T}\right)$, is $\left\{\lambda_{1}, \ldots, \lambda_{k-1}, \mu_{k}, \lambda_{k+1}, \ldots, \lambda_{n}\right\}$ ?

The following result answers this question. 
Proposition 3 Consider a pair $(A, b)$, let $\sigma(A)=\left\{\lambda_{1}, \lambda_{2}, \ldots, \lambda_{n}\right\}$ and let $x_{k}$ be an eigenvector of $A^{T}$ associated with $\lambda_{k}$. If $b^{T} x_{k} \neq 0$, then there exists a vector $f$ such that $\sigma\left(A+b f^{T}\right)=\left\{\lambda_{1}, \ldots, \lambda_{k-1}, \mu_{k}, \lambda_{k+1}, \ldots, \lambda_{n}\right\}$.

Proof: As $\sigma\left(A^{T}\right)=\sigma(A)$, by Brauer's Theorem 1 applied to $A^{T}$, the matrix $A^{T}+x_{k} q^{T}$ has eigenvalues $\lambda_{1}, \ldots, \lambda_{k-1}, \lambda_{k}+q^{T} x_{k}, \lambda_{k+1}, \ldots, \lambda_{n}$, where $q$ is any $n$-dimensional vector. It is clear that $\sigma\left(A+q x_{k}^{T}\right)=\left\{\lambda_{1}, \ldots, \lambda_{k-1}, \lambda_{k}+q^{T} x_{k}\right.$, $\left.\lambda_{k+1}, \ldots, \lambda_{n}\right\}$.

Consider $q=b$ and $f=x_{k}$. If $b^{T} x_{k} \neq 0$, we have:

$$
\lambda_{k}+q^{T} x_{k}=\lambda_{k}+b^{T} x_{k}=\mu_{k} \quad \Longrightarrow \quad b^{T} x_{k}=\mu_{k}-\lambda_{k},
$$

then $\sigma\left(A+b f^{T}\right)=\left\{\lambda_{1}, \ldots, \lambda_{k-1}, \lambda_{k}+q^{T} x_{k}, \lambda_{k+1}, \ldots, \lambda_{n}\right\}$.

Remark 2 (a) Note that the assumption of $b^{T} x_{k} \neq 0$ is needed only to assure the change of the eigenvalue $\lambda_{k}$. Otherwise no eigenvalue changes.

(b) By this result we can say that the pole assignment problem has a solution if $x_{k}$ is not orthogonal to the vector $b$ (that is, $b^{T} x_{k} \neq 0$ ) (see [2]). When this condition holds for all eigenvectors of $A^{T}$, then the pair $(A, b)$ is called completely controllable, in this case the solution is unique [3].

(c) According to Proposition 1 the eigenvector of $A^{T}$ associated with $\lambda_{k}$ and the eigenvectors of $A^{T}$ corresponding to $\lambda_{i}, i \neq k$, such that $b^{T} x_{i}=0$ remain unchanged.

(d) If $\lambda_{i} \neq \lambda_{j}$ for each $i \neq j$, and $b^{T} x_{i} \neq 0$, then on can show that $b^{T} w_{i} \neq 0$, where $w_{i}$ is defined in Proposition 1.

Example 1 Consider the pair $(A, b)$ :

$$
A=\left[\begin{array}{rrrr}
-2 & -3 & -2 & 0 \\
2 & 3 & 2 & 0 \\
3 & 3 & 3 & 0 \\
0 & 1 & -2 & 2
\end{array}\right], \quad b=\left[\begin{array}{l}
0 \\
0 \\
1 \\
1
\end{array}\right]
$$

This pair $(A, b)$ is not completely controllable since the rank of the controllability matrix

$$
\mathcal{C}(A, b)=\left[\begin{array}{llll}
b & A b & A^{2} b & A^{3} b
\end{array}\right]=\left[\begin{array}{rrrr}
0 & -2 & -8 & -26 \\
0 & 2 & 8 & -26 \\
1 & 3 & 9 & 27 \\
1 & 0 & -4 & -18
\end{array}\right]
$$

is 3. Note that $\sigma(A)=\sigma\left(A^{T}\right)=\{0,1,2,3\}$ and the eigenvectors of $A^{T}$ are:

$$
\begin{aligned}
& x_{\lambda=0}^{T}=\left(\alpha_{1},-\alpha_{1}, 0,0\right) \quad \forall \alpha_{1} \neq 0 \quad \Longrightarrow \quad b^{T} x_{\lambda=0}=0 \\
& x_{\lambda=1}^{T}=\left(\alpha_{2}, 0, \alpha_{2}, 0\right) \quad \forall \alpha_{2} \neq 0 \quad \Longrightarrow \quad b^{T} x_{\lambda=1}=\alpha_{2} \\
& x_{\lambda=2}^{T}=\left(\alpha_{3}, 2 \alpha_{3}, 0, \alpha_{3}\right) \quad \forall \alpha_{3} \neq 0 \quad \Longrightarrow \quad b^{T} x_{\lambda=2}=\alpha_{3} \\
& x_{\lambda=3}^{T}=\left(\alpha_{4}, \alpha_{4}, \alpha_{4}, 0\right) \quad \forall \alpha_{4} \neq 0 \quad \Longrightarrow \quad b^{T} x_{\lambda=3}=\alpha_{4}
\end{aligned}
$$


Although the system is not completely controllable, we can change all the eigenvalues of $A$, but $\lambda=0$. For instance, if we change $\lambda=3$ to $\mu=0.7$ and consider the eigenvector of $A^{T}$ associated with $\lambda=3$, we obtain

$$
b^{T} x_{\lambda=3}=\alpha_{4}=0.7-3=-2.3 \quad \Longrightarrow \quad \alpha_{4}=-2.3 .
$$

Then, $f^{T}=(-2.3,-2.3,-2.3,0)$ and

$$
A+b f^{T}=\left[\begin{array}{rrrr}
-2 & -3 & -2 & 0 \\
2 & 3 & 2 & 0 \\
0.7 & 0.7 & 0.7 & 0 \\
-2.3 & -1.3 & -4.3 & 2
\end{array}\right] \text { with } \sigma\left(A+b f^{T}\right)=\{0,0.7,1,2\}
$$

Consider a SISO discrete-time (or continuous-time) invariant linear system given by the pair $\left(A^{T}, b\right)$. Let $\sigma\left(A^{T}\right)=\left\{\lambda_{1}, \lambda_{2}, \ldots, \lambda_{n}\right\}$. The system is asymptotically stable if all eigenvalues $\lambda_{i}$ of $A^{T}$ satisfy $\left|\lambda_{i}\right|<1$ (or $\operatorname{Re}\left(\lambda_{i}\right)<0$ ), see for instance $[3,5]$. Applying Proposition 3 to an unstable pair $(A, b)$ we can obtain the closed-loop system $A+b f^{T}$ with the feedback vector $f$ equal to the eigenvector associated with the eigenvalue $\lambda_{k}$ such that $\left|\lambda_{k}\right| \geq 1\left(\operatorname{or} \operatorname{Re}\left(\lambda_{k}\right) \geq 0\right)$.

The following algorithm gives a verification of stabilization of the SISO system $\left(A^{T}, b\right)$ with application of Proposition 3 and the Power Method [8] assuming that $A^{T}$ has a dominant eigenvalue. The advantage of the proposed method is that we do not need the system to be completely controllable.

Algorithm Input: $\left(A^{T}, b\right)$.

Step 1. Set $A_{0}=A_{1}=A, i=1$ and $f_{0}$ the zero vector.

Step 2. Apply the Power Method to $A_{i}$, and obtain the dominant eigenvalue $\lambda_{i}$ and the corresponding eigenvector $x_{i}$.

Step 3. If $\left|\lambda_{i}\right|<1$, then the pair $\left(A_{i}, b\right)$ is asymptotically stable, where $A_{i}=$ $A_{i-1}+f_{i-1} b^{T}$. END.

Otherwise,

Step 4. If $\left\langle x_{i}, b\right\rangle=0$, then the pair $\left(A_{i}, b\right)$ cannot be stabilized (Proposition 3 ) END.

Otherwise,

Step 5. Choose a scalar $\alpha_{i}$ such that the new eigenvalue $\mu_{i}=\lambda_{i}+\left(\alpha_{i} x_{i}^{T}\right) b$ satisfies $\left|\mu_{i}\right|<1$. Let $f_{i}=f_{i-1}+\alpha_{i} x_{i}$.

Step 6. Let $A_{i+1}=A_{i}+\alpha_{i} x_{i} b^{T}$. Note that $\sigma\left(A_{i+1}\right)=\left\{\lambda_{1}, \ldots, \lambda_{i-1}, \mu_{i}, \lambda_{i+1}\right.$, $\left.\ldots, \lambda_{n}\right\}$ with $\left|\mu_{i}\right|<1$. Let $i=i+1$, GOTO Step 2. 


\section{Rado's theorem}

Perfect [7] in 1955 presented the following result, due to R. Rado, which shows how to modify, in only one step, $r$ eigenvalues of an arbitrary matrix $A$ without changing any of the remaining $n-r$ eigenvalues. Rado's Theorem is an extension of Brauer's Theorem and it has been applied to generate sufficient conditions for the construction of nonnegative matrices with prescribed spectrum [7, 9]. As in the previous case, the immediate consequences of this result are the block deflation methods and the pole assignment problem when the MIMO linear control system is not completely controllable.

Theorem 2 [9, Brauer's Extended Theorem, Theorem 5] Let $A$ be an arbitrary $n \times n$ matrix with eigenvalues $\left\{\lambda_{1}, \lambda_{2}, \ldots, \lambda_{n}\right\}$. Let $X=\left[x_{1} x_{2} \ldots x_{r}\right]$ be an $n \times r$ matrix such that $\operatorname{rank}(X)=r$ and $A x_{i}=\lambda_{i} x_{i}, i=1,2, \ldots, r, r \leq n$. Let $C$ be an arbitrary $r \times n$ matrix. Then the matrix $A+X C$ has eigenvalues $\left\{\mu_{1}, \mu_{2}, \ldots, \mu_{r}, \lambda_{r+1}, \lambda_{r+2}, \ldots, \lambda_{n}\right\}$, where $\mu_{1}, \mu_{2}, \ldots, \mu_{r}$ are eigenvalues of the matrix $\Omega+C X$ with $\Omega=\operatorname{diag}\left(\lambda_{1}, \lambda_{2}, \ldots, \lambda_{r}\right)$.

Theorem 2 shows how to change $r$ eigenvalues of $A$ in only one step. In general, the eigenvector $x_{i}$ associated with $\lambda_{i}$ of $A, i=1,2, \ldots, r$, is not the eigenvector associated with the new eigenvalue $\mu_{i}$ of $A+X C$. If the matrix $\Omega+C X$ is diagonalizable the way in which $x_{i}$ changes is described below.

Proposition 4 Let $A$ be an arbitrary $n \times n$ matrix with eigenvalues $\left\{\lambda_{1}, \lambda_{2}\right.$, $\left.\ldots, \lambda_{n}\right\}$. Let $X=\left[x_{1} x_{2} \ldots x_{r}\right]$ be an $n \times r$ matrix which column vectors satisfy $A x_{i}=\lambda_{i} x_{i}, i=1,2, \ldots, r, r \leq n$. Let $C$ be an arbitrary $r \times n$ matrix and let $\Omega=\operatorname{diag}\left(\lambda_{1}, \lambda_{2}, \ldots, \lambda_{r}\right)$.

If $\mu_{1}, \mu_{2}, \ldots, \mu_{r}$ are eigenvalues of the diagonalizable matrix $\Omega+C X$ and $T$ is the transition matrix to its Jordan form, then the column vectors of the matrix product $X T$ are the eigenvectors of $A+X C$ associated with $\mu_{1}, \mu_{2}, \ldots, \mu_{r}$.

Proof: Since $T$ is the transition matrix, we have

$$
(A+X C) X=X(\Omega+C X)=X T \operatorname{diag}\left(\mu_{1}, \mu_{2}, \ldots, \mu_{r}\right) T^{-1} .
$$

Hence $(A+X C) X T=X T \operatorname{diag}\left(\mu_{1}, \mu_{2}, \ldots, \mu_{r}\right)$ and the result follows.

Remark 3 If we take an arbitrary matrix $C$ such that

$$
C X=\operatorname{diag}\left(\mu_{1}-\lambda_{1}, \mu_{2}-\lambda_{2}, \ldots, \mu_{r}-\lambda_{r}\right)
$$

then $\Omega+C X=\operatorname{diag}\left(\mu_{1}, \mu_{2}, \ldots, \mu_{r}\right)$, and the matrix $T$, of Proposition 4 , is equal to the identity matrix. Therefore, the eigenvector $x_{i}$ associated with $\lambda_{i}$ of $A, i=1,2, \ldots, r$, is the eigenvector associated with the new eigenvalue $\mu_{i}$ of $A+X C$.

In this case, the eigenvectors associated with the eigenvalues $\lambda_{r+1}, \ldots, \lambda_{n}$ change in the following way. 
Proposition 5 Assume the assumptions of Theorem 2 and Remark 3 hold. Let $x_{i}$ be the eigenvector of $A$ associated with the eigenvalue $\lambda_{i}, r+1 \leq i \leq n$. Then, the eigenvector of $A+X C$ associated with $\lambda_{i}$ is given by:

$$
w_{i}=x_{i}-\sum_{j=1}^{r} \frac{c_{j} x_{i}}{\mu_{j}-\lambda_{i}} x_{j}, \quad r+1 \leq i \leq n,
$$

where $c_{j}$ is the $j$ th row of the matrix $C$.

Proof: For $x_{i}, r+1 \leq i \leq n$, we have

$$
\begin{aligned}
& (A+X C)\left(x_{i}-\sum_{j=1}^{r} \frac{c_{j} x_{i}}{\mu_{j}-\lambda_{i}} x_{j}\right)=A x_{i}+X C x_{i}-\sum_{j=1}^{r}(A+X C) \frac{c_{j} x_{i}}{\mu_{j}-\lambda_{i}} x_{j} \\
& =\lambda_{i} x_{i}+\sum_{j=1}^{r}\left(c_{j} x_{i}\right) x_{j}-\sum_{j=1}^{r} \frac{c_{j} x_{i}}{\mu_{j}-\lambda_{i}} \mu_{j} x_{j} \\
& =\lambda_{i} x_{i}-\sum_{j=1}^{r}\left(-\left(c_{j} x_{i}\right)+\frac{c_{j} x_{i}}{\mu_{j}-\lambda_{i}} \mu_{j}\right) x_{j} \\
& =\lambda_{i}\left(x_{i}-\sum_{j=1}^{r} \frac{c_{j} x_{i}}{\mu_{j}-\lambda_{i}}\right) x_{j} .
\end{aligned}
$$

\section{Applications of Rado's Theorem}

In this section we give applications of Rado's Theorem to deflation techniques and to the pole assignment problem for MIMO systems.

\subsection{Block deflation techniques}

Now using Rado's Theorem 2 we can obtain a block version of the deflation results working with particular matrices $C$. A direct application of Rado's Theorem gives

Corollary 3 (Wielandt's deflation) Assume assumptions of Theorem 2 hold. Let $C$ be a matrix such that $\Omega+C X$ has all the eigenvalues zero. Then the matrix $B=A+X C$ has eigenvalues $\left\{0,0, \ldots, 0, \lambda_{r+1}, \lambda_{r+2}, \ldots, \lambda_{n}\right\}$.

Remark 4 If $A$ is symmetric, then it is diagonalizable and we can choose an orthogonal matrix $X=\left[x_{1} \ldots x_{r} x_{r+1} \ldots x_{n}\right]=\left[X_{r} X_{n-r}\right]$ made of eigenvectors of $A$. Consider $\Theta=\operatorname{diag}\left(\mu_{1}-\lambda_{1}, \mu_{2}-\lambda_{2}, \ldots, \mu_{r}-\lambda_{r}\right)$, then the matrix 
$B=A+X_{r} \Theta X_{r}^{T}$ is symmetric (diagonalizable) and it can be verified that its eigenvectors associated with the eigenvalues $\lambda_{r+1}, \ldots, \lambda_{n}$ are the eigenvectors of $A$.

Corollary 4 (Hotelling's deflation) Assume assumptions of Theorem 2 hold. (i) (Symmetric case.) Let $A$ be symmetric. Then the symmetric matrix $A-$ $X \Omega X^{T}$ has the eigenvalues $\left\{0,0, \ldots, 0, \lambda_{r+1}, \lambda_{r+2}, \ldots, \lambda_{n}\right\}$, provided that $X^{T} X=I_{r}$.

(ii) (Nonsymmetric case.) Let $L=\left[l_{1}, l_{2}, \ldots, l_{r}\right]$ be an $n \times r$ matrix such that $\operatorname{rank}(L)=r, l_{i}^{T} A=\lambda_{i} l_{i}^{T}$ and $L^{T} X=I$. Then the matrix $B=A-X \Omega L^{T}$ has eigenvalues $\left\{0,0, \ldots, 0, \lambda_{r+1}, \lambda_{r+2}, \ldots, \lambda_{n}\right\}$.

Proof: Apply Rado's Theorem with $C=-\Omega X^{T}$ for the symmetric case and with $C=-\Omega L^{T}$ for the nonsymmetric case.

Remark 5 It is easy to check that the matrices $A$ and $A+X C$ have the same eigenvectors and the same Jordan structure associated with the eigenvalues $\lambda_{r+1}, \lambda_{r+2}, \ldots, \lambda_{n}$

\subsection{Pole assignment of MIMO systems}

An immediate application of Rado's Theorem 2 to control theory in multiinput, multi-output (MIMO) systems defined by the pair $(A, B)$ is the following problem, where we assume that the new eigenvalues $\mu_{i}$ are different from the eigenvalues to be changed $\lambda_{j}, 1 \leq i, j \leq r$.

Consider a pair $(A, B)$ with $A$ and $B n \times n$ and $n \times m$ matrices and the set of numbers $\left\{\mu_{1}, \mu_{2}, \ldots, \mu_{r}\right\}$, and let $\sigma(A)=\left\{\lambda_{1}, \lambda_{2}, \ldots, \lambda_{n}\right\}$. What are the conditions on $(A, B)$ so that the spectrum of the closed loop matrix $A+B F^{T}, \sigma\left(A+B F^{T}\right)$, coincides with the set $\left\{\mu_{1}, \mu_{2}, \ldots\right.$, $\left.\mu_{r}, \lambda_{r+1}, \lambda_{r+2}, \ldots, \lambda_{n}\right\}$, for some matrix $F$ ?

The following result answers this question.

Proposition 6 Consider a pair $(A, B)$, with $A$ and $B n \times n$ and $n \times m$ matrices. Let $\sigma(A)=\left\{\lambda_{1}, \lambda_{2}, \ldots, \lambda_{n}\right\}$. Let $X=\left[x_{1} x_{2} \ldots x_{r}\right]$ be an $n \times r$ matrix such that $\operatorname{rank}(X)=r$ and $A^{T} x_{i}=\lambda_{i} x_{i}, i=1,2, \ldots, r, r \leq n$. If there is a column $b_{j_{i}}$ of the matrix $B$ such that $b_{j_{i}}^{T} x_{i} \neq 0$, for all $i=1,2, \ldots, r$, then there exists a matrix $F$ such that $\sigma\left(A+B F^{T}\right)=\left\{\mu_{1}, \mu_{2}, \ldots, \mu_{r}, \lambda_{r+1}, \lambda_{r+2}, \ldots, \lambda_{n}\right\}$.

Proof: As $\sigma\left(A^{T}\right)=\sigma(A)$, by Rado's Theorem 2 applied to $A^{T}$, we have that $\sigma\left(A^{T}+X C\right)=\left\{\mu_{1}, \mu_{2}, \ldots, \mu_{r}, \lambda_{r+1}, \lambda_{r+2}, \ldots, \lambda_{n}\right\}$, where $\left\{\mu_{1}, \mu_{2}, \ldots, \mu_{r}\right\}$ are the eigenvalues of $\Omega+C X$, with $\Omega=\operatorname{diag}\left(\lambda_{1}, \lambda_{2}, \ldots, \lambda_{r}\right)$. Then, $\sigma\left(A+C^{T} X^{T}\right)=$ $\left\{\mu_{1}, \mu_{2}, \ldots, \mu_{r}, \lambda_{r+1}, \lambda_{r+2}, \ldots, \lambda_{n}\right\}$.

Let $C^{T}=\left[\begin{array}{llll}b_{j_{1}} & b_{j_{2}} & \ldots & b_{j_{r}}\end{array}\right]$, where $b_{j_{i}}^{T} x_{i} \neq 0$ for $i=1,2, \ldots, r$. Then

$$
A+C^{T} X^{T}=A+\left[\begin{array}{lllll}
b_{j_{1}} & b_{j_{2}} & \ldots & b_{j_{r}}
\end{array}\right] X^{T}=A+B\left[\begin{array}{llll}
e_{j_{1}} & e_{j_{2}} & \ldots & e_{j_{r}}
\end{array}\right] X^{T},
$$


where the matrix $\left[\begin{array}{llll}e_{j_{1}} & e_{j_{2}} & \ldots & e_{j_{r}}\end{array}\right]$ is made of the corresponding unit vectors. Setting $F^{T}=\left[\begin{array}{llll}e_{j_{1}} & e_{j_{2}} & \cdots & e_{j_{r}}\end{array}\right] X^{T}$, we have

$$
\sigma\left(A+C^{T} X^{T}\right)=\sigma\left(A+B F^{T}\right)=\left\{\mu_{1}, \mu_{2}, \ldots, \mu_{r}, \lambda_{r+1}, \lambda_{r+2}, \ldots, \lambda_{n}\right\},
$$

where $\left\{\mu_{1}, \mu_{2}, \ldots, \mu_{r}\right\}$ are the eigenvalues of $\Omega+\left[\begin{array}{llll}e_{j_{1}} & e_{j_{2}} & \ldots & e_{j_{r}}\end{array}\right]^{T} B^{T} X$, with $\Omega=\operatorname{diag}\left(\lambda_{1}, \lambda_{2}, \ldots, \lambda_{r}\right)$.

Remark 6 (a) Note that the assumption of existence of a column $b_{j_{i}}$ of the matrix $B$ such that $b_{j_{i}}^{T} x_{i} \neq 0$, for $i=1,2, \ldots, r$, is needed only to assure the change of the eigenvalue $\lambda_{i}$. Otherwise no eigenvalue changes.

(b) In the MIMO systems the solution of the pole assignment is not unique as we can see in the next example. Further, note that Proposition 6 indicates that we can allocate poles even in the case of uncontrollable systems.

Example 2 Consider the pair $(A, B)$ :

$$
A=\left[\begin{array}{rrrr}
-2 & -3 & -2 & 0 \\
2 & 3 & 2 & 0 \\
3 & 3 & 3 & 0 \\
0 & 1 & -2 & 2
\end{array}\right], \quad B=\left[\begin{array}{ll}
0 & 0 \\
0 & 0 \\
1 & 1 \\
1 & 1
\end{array}\right]
$$

Note that this pair is not completely controlable since the rank of the matrix

$$
\mathcal{C}(A, B)=\left[\begin{array}{llll}
B & A B & A^{2} B & A^{3} B
\end{array}\right]=\left[\begin{array}{rrrrrrrr}
0 & 0 & -2 & -2 & -8 & -8 & -26 & -26 \\
0 & 0 & 2 & 2 & 8 & 8 & -26 & -26 \\
1 & 1 & 3 & 3 & 9 & 9 & 27 & 27 \\
1 & 1 & 0 & 0 & -4 & -4 & -18 & -18
\end{array}\right]
$$

is 3. The spectral computation gives $\sigma(A)=\sigma\left(A^{T}\right)=\{0,1,2,3\}$ and the eigenvectors of $A^{T}$ are:

$$
\begin{aligned}
& x_{\lambda=0}^{T}=\left(\alpha_{1},-\alpha_{1}, 0,0\right) \quad \forall \alpha_{1} \neq 0 \quad \Longrightarrow \quad B^{T} x_{\lambda=0}=\left[\begin{array}{l}
0 \\
0
\end{array}\right] \\
& x_{\lambda=1}^{T}=\left(\alpha_{2}, 0, \alpha_{2}, 0\right) \quad \forall \alpha_{2} \neq 0 \quad \Longrightarrow \quad B^{T} x_{\lambda=1}=\left[\begin{array}{c}
\alpha_{2} \\
\alpha_{2}
\end{array}\right] \\
& x_{\lambda=2}^{T}=\left(\alpha_{3}, 2 \alpha_{3}, 0, \alpha_{3}\right) \quad \forall \alpha_{3} \neq 0 \quad \Longrightarrow \quad B^{T} x_{\lambda=2}=\left[\begin{array}{c}
\alpha_{3} \\
\alpha_{3}
\end{array}\right] \\
& x_{\lambda=3}^{T}=\left(\alpha_{4}, \alpha_{4}, \alpha_{4}, 0\right) \quad \forall \alpha_{4} \neq 0 \quad \Longrightarrow \quad B^{T} x_{\lambda=3}=\left[\begin{array}{c}
\alpha_{4} \\
\alpha_{4}
\end{array}\right]
\end{aligned}
$$

Since the above products are different from zero for the eigenvalues $\lambda=1, \lambda=2$ and $\lambda=3$, we consider three cases according to the number of eigenvalues we want to change and the number of columns of the matrix $B$.

Case 1. Suppose we want to change the eigenvalues $\lambda=2$ and $\lambda=3$ to $\mu=0.5$ and $\mu=0.7$, respectively. Then, $r=m$. Since $b_{1}^{T} x_{\lambda=2} \neq 0$ and $b_{1}^{T} x_{\lambda=3} \neq 0$,

$$
C^{T}=\left[b_{1} b_{1}\right]=B\left[\begin{array}{ll}
1 & 1 \\
0 & 0
\end{array}\right]
$$


and the matrix

$$
\Omega+C X=\Omega+\left[\begin{array}{ll}
1 & 0 \\
1 & 0
\end{array}\right] B^{T} X=\left[\begin{array}{cc}
2+\alpha_{3} & \alpha_{4} \\
\alpha_{3} & 3+\alpha_{4}
\end{array}\right]
$$

has the eigenvalues $\mu_{1}=0.5$ and $\mu_{2}=0.7$ when $\alpha_{3}=1.95$ and $\alpha_{4}=-5.75$. So the feedback matrix $F$ is

$$
F^{T}=\left[\begin{array}{ll}
1 & 1 \\
0 & 0
\end{array}\right] X^{T}=\left[\begin{array}{cccc}
-3.8 & -1.85 & -5.75 & 1.95 \\
0 & 0 & 0 & 0
\end{array}\right] .
$$

Then, the closed-loop matrix

$$
A+B F^{T}=\left[\begin{array}{cccc}
-2 & -3 & -2 & 0 \\
2 & 3 & 2 & 0 \\
-0.8 & 1.15 & -2.75 & 1.95 \\
-3.8 & -0.85 & -7.75 & 3.95
\end{array}\right]
$$

has the spectrum $\sigma\left(A+B F^{T}\right)=\{0,0.5,0.7,1\}$.

Note that working with the two column vectors of the matrix $B$, we obtain the feedback matrix

$$
F^{T}=\left[\begin{array}{cccc}
1.95 & 3.9 & 0 & 1.95 \\
-5.75 & -5.75 & -5.75 & 0
\end{array}\right] .
$$

Case 2. Now, we want to change only the eigenvalue $\lambda=3$ to $\mu=0.7$, in this case $r<m$. Since $b_{1}^{T} x_{\lambda=3} \neq 0$,

$$
C^{T}=\left[b_{1}\right]=B\left[\begin{array}{l}
1 \\
0
\end{array}\right]
$$

and the matrix $\Omega+C X=\Omega+[10] B^{T} X=3+\alpha_{4}$ has the eigenvalue $\mu=0.7$ if $\alpha_{4}=-2.3$. So the feedback matrix $F$ is

$$
F^{T}=\left[\begin{array}{l}
1 \\
0
\end{array}\right] X^{T}=\left[\begin{array}{cccc}
-2.3 & -2.3 & -2.3 & 0 \\
0 & 0 & 0 & 0
\end{array}\right] .
$$

Then, the closed-loop matrix is

$$
A+B F^{T}=\left[\begin{array}{rrrr}
-2 & -3 & -2 & 0 \\
2 & 3 & 2 & 0 \\
0.7 & 0.7 & 0.7 & 0 \\
-2.3 & -1.3 & -4.3 & 2
\end{array}\right]
$$

with the spectrum $\sigma\left(A+B F^{T}\right)=\{0,0.7,1,2\}$.

Case 3. Finally, we want to change the three eigenvalues $\lambda=1, \lambda=2$ and $\lambda=3$ to $\mu_{1}=0.2, \mu_{2}=0.5$ and $\mu_{3}=0.7$, respectively. In this case $r>m$.

Since $b_{1}^{T} x_{\lambda=1} \neq 0, b_{1}^{T} x_{\lambda=2} \neq 0$ and $b_{1}^{T} x_{\lambda=3} \neq 0$,

$$
C^{T}=\left[b_{1} b_{1} b_{1}\right]=B\left[\begin{array}{lll}
1 & 1 & 1 \\
0 & 0 & 0
\end{array}\right]
$$


and the matrix

$$
\Omega+C X=\Omega+\left[\begin{array}{ll}
1 & 0 \\
1 & 0 \\
1 & 0
\end{array}\right] B^{T} X=\left[\begin{array}{ccc}
1+\alpha_{2} & \alpha_{3} & \alpha_{4} \\
\alpha_{2} & 2+\alpha_{3} & \alpha_{4} \\
\alpha_{2} & \alpha_{3} & 3+\alpha_{4}
\end{array}\right]
$$

has eigenvalues $\mu_{1}=0.2, \mu_{2}=0.5$ and $\mu_{3}=0.7$ when $\alpha_{2}=-0.06, \alpha_{3}=3.51$ and $\alpha_{4}=-8.05$. So the feedback matrix $F$ is

$$
F^{T}=\left[\begin{array}{lll}
1 & 1 & 1 \\
0 & 0 & 0
\end{array}\right] X^{T}=\left[\begin{array}{cccc}
-4.6 & -1.03 & -8.11 & 3.51 \\
0 & 0 & 0 & 0
\end{array}\right] .
$$

Then, the closed-loop matrix is

$$
A+B F^{T}=\left[\begin{array}{rrrr}
-2 & -3 & -2 & 0 \\
2 & 3 & 2 & 0 \\
-1.6 & 1.97 & -5.11 & 3.51 \\
-4.6 & -0.03 & -10.11 & 5.51
\end{array}\right]
$$

with the spectrum $\sigma\left(A+B F^{T}\right)=\{0,0.2,0.5,0.7\}$.

Remark 7 As before a MIMO discrete-time (or continuous-time) invariant linear system, given by the pair $\left(A^{T}, B\right)$, is asymptotically stable if all eigenvalues $\lambda_{i}$ of $A^{T}$ satisfy $\left|\lambda_{i}\right|<1$ (or $\operatorname{Re}\left(\lambda_{i}\right)<0$ ), see for instance [3, 5]. Applying Proposition 6 to an unstable pair $\left(A^{T}, B\right)$ we can obtain the closed-loop system $A+B F^{T}$ with the feedback matrix $F$ computed as in the proof of the above proposition.

\section{Acknowledgements}

This work is supported by Fondecyt 1085125, Chile, the Spanish grant DGI MTM2010-18228 and the Programa de Apoyo a la Investigación y Desarrollo (PAID-06-10) of the UPV.

\section{References}

[1] A. Brauer. Limits for the characteristic roots of matrices IV: Applications to stochastic matrices. Duke Math. J., vol. 19 (1952), 75-91.

[2] P. E. Crouch. Introduction to Mathematical Systems Theory. Lecture notes for a joint course at the Universities of Warwick and Bremen. Control Theory Centre Publication. Forschungsschwerpunkt Dynamische Systeme, 1983.

[3] D. F. Delchamps. State-Space and Input-Output Linear Systems, SpringerVerlag, New York, 1988.

[4] M. L. J. Hautus. Controllability and observability condition of linear autonomous systems. Ned. Akad. Wetenschappen, Proc. Ser. A, vol. 72, (1969), 443-448. 
[5] T. Kailath, Linear Systems, Prentice-Hall, Englewood Cliffs, 1980.

[6] A. N. Langville, C. D. Meyer. Deeper Inside PageRank. Internet Mathematics, vol. 1 (2004), 1-33.

[7] H. Perfect. Methods of constructing certain stochastic matrices. II. Duke Math. J., vol. 22 (1955), 305-311.

[8] Y. Saad. Numerical Methods for large eigenvalue problems, SIAM, Philadelphia, 2011.

[9] R. L. Soto, O. Rojo. Applications of a Brauer theorem in the nonnegative inverse eigenvalue problem. Linear Algebra and its Applications, vol. 416 (2006), 844-856.

[10] J. H. Wilkinson, The Algebraic Eigenvalue Problem, Oxford Science, Oxford, England, 1965. 